\title{
Lattice QCD Studies of Pentaquarks and Exotics
}

B. G. Lasscock* a , J. Hedditch ${ }^{\mathrm{a}}$, W. Kamleh ${ }^{\mathrm{b}}$, D. B. Leinweber ${ }^{\mathrm{a}}$, W. Melnitchouk ${ }^{\mathrm{c}}$, A. W. Thomas ${ }^{\mathrm{c}}$, A. G. Williams ${ }^{\text {a† }}$, R. D. Young ${ }^{\mathrm{c}}$, J. M. Zanotti ${ }^{\mathrm{d}}$

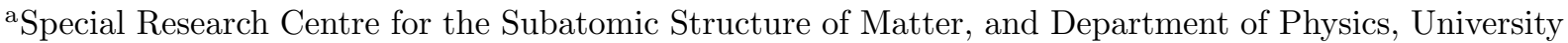
of Adelaide, Adelaide SA 5005, Australia

${ }^{\mathrm{b}}$ School of Mathematics, Trinity College, Dublin 2, Ireland

${ }^{\mathrm{c}}$ Jefferson Lab, 12000 Jefferson Ave., Newport News, VA 23606 USA

${ }^{\mathrm{d}}$ School of Physics, University of Edinburgh, Edinburgh EH9 3JZ, UK

The possible discovery of the $\Theta^{+}$pentaquark has motivated a number of studies into its nature using lattice QCD. Initial studies focused on spin- $1 / 2$ states and more recently also spin- $3 / 2$ states. Here we report the results of the first exploratory study in quenched lattice QCD of pentaquarks with both spin- $1 / 2$ and spin- $3 / 2$ using the FLIC fermion action. We do not find any evidence for the standard lattice resonance signature of attraction (i.e. binding at quark masses near the physical regime) in the spin- $1 / 2$ channels or in the $J^{P}=3 / 2^{-}$channel. Some evidence of binding is inferred in the isoscalar $3 / 2^{+}$channel. We also present the results of our study into exotic meson states using hybrid meson interpolators with explicit gluonic degrees of freedom. We find a candidate for the $J^{P C}=1^{-+}$exotic meson which has a mass consistent with the $\pi_{1}(1600)$ experimental candidate.

\section{Introduction}

We present the results of our search for the putative $\Theta^{+}$pentaquark and $J^{P C}=1^{-+}$exotic meson in lattice QCD. The $\Theta^{+}$pentaquark would be an exotic baryon, meaning its quantum numbers cannot be described by a configuration of threequarks alone. The candidate $\Theta^{+}$has been reported to have a mass of $1540 \mathrm{MeV}$, strangeness +1 and minimal quark content uudds; its spin, parity and isospin are not claimed to be known [1]. Since the spin, parity and isospin of the candidate $\Theta^{+}$remain unknown we explore an exhaustive array of local pentaquark interpolating fields $[2,3]$, including the first exploratory study into the $\Theta^{+}$as a spin-3/2 state [3], to search for evidence of the $\Theta^{+}$pentaquark.

Similarly exotic mesons have quantum num-

\footnotetext{
* On behalf of the collaboration, the author thanks the Australian partnership for advanced computing (APAC) national facility for the computer time that made this study possible, and for further support from the South Australian partnership for advanced computing (SAPAC). †'Speaker
}

bers that cannot be described in terms of a simple quark-antiquark pair. In our study we access exotic meson states using hybrid interpolating fields, which have explicit gluonic degrees of freedom. The particle data group [4] has reported two candidates for the $1^{-+}$exotic at $1400 \mathrm{MeV}$ and $1600 \mathrm{MeV}$. Further discussion of the experimental status of the $1^{-+}$exotic can be found in Refs. $[5,6]$. We find a $1^{-+}$state appareently consistent with the $1600 \mathrm{MeV}$ candidate [7].

Central to this work is the formulation of a signature that distinguishes a resonance from possible two-particle states with the same quantum numbers. A number of such techniques have been developed recently in the search for evidence of the $\Theta^{+}$pentaquark in lattice QCD. The volume dependence of the residue of the lowest lying state has been proposed as a way to identify a resonance $[8,9]$. Alternatively, hybrid boundary conditions, i.e. choosing a different boundary condition for the $u$ and $d$ quarks compared to the $s$ quark, have been proposed in Refs. [10-12]. Our method is complementary to these approaches. In 
Refs. $[2,3]$ we look for evidence of attraction between the constituents of the resonance such that the mass of the state becomes lower than the free decay channel energy at quark masses near the physical regime. This resonance signature is universally observed in lattice QCD studies of the nucleon resonances [13-17]. We find evidence of this signature for the $\Theta^{+}$in the spin-3/2 positive parity channel. Further work in this channel is warranted to determine if this evidence demands the existence of a spin-3/2 pentaquark [3] .

In Sec. 2 we review the standard techniques for extracting hadron masses in lattice QCD and state our lattice simulation parameters. The lattice resonance signature is discussed in Sec. 3. In Sec. 4 we present our results for the $1^{-+}$meson and the spin- $1 / 2$ and spin- $3 / 2$ pentaquark states, and in Sec. 5 we conclude that there exists a potential candidate for a $I\left(J^{P}\right)=0\left(3 / 2^{+}\right)$pentaquark and a signal consistent with the $\pi_{1}(1600)$ $1^{-+}$exotic meson.

\section{Lattice details}

\subsection{Lattice techniques}

In lattice $\mathrm{QCD}$ we derive hadron masses from the 2-pt correlation function,

$$
\mathcal{G}(t, \vec{p})=\sum_{\vec{x}} \exp (-i \vec{p} \cdot \vec{x})\langle 0|T \chi(x) \bar{\chi}(0)| 0\rangle .
$$

The time ordered product of fields shown above can be expressed in terms of quark propagators, allowing us to evaluate this function. Here the interpolating fields $\bar{\chi},(\chi)$ create (annihilate) states with a particular set of quantum numbers. When studying baryon correlation functions, we must project out states of the appropriate spin and parity using the projection operator, $\Gamma[15]$. We extract the energy of the lowest lying state, with energy $E_{0}$, created by our interpolator using,

$$
G(t, \vec{p}) \underset{\substack{t \rightarrow \infty \\ \propto}}{=} \operatorname{tr}_{\mathrm{sp}}[\Gamma \mathcal{G}(t, \vec{p})],
$$

Since the contributions to the two-point function are exponentially suppressed at a rate proportional to the energy of the state, at zero momentum the mass of the lightest state, $m_{0}$, is obtained by fitting a constant to the effective mass,

$$
\begin{aligned}
M^{\mathrm{eff}}(t) & =\ln \left(\frac{G(t, \overrightarrow{0})}{G(t+1, \overrightarrow{0})}\right) \\
& \stackrel{t \rightarrow \infty}{=} m_{0} .
\end{aligned}
$$

\subsection{Interpolating fields}

In this section we review the interpolating fields that we use in our studies of exotic hadrons.

The two general types of pentaquark interpolating fields we consider are those based on an " $N K$ " configuration (either $n K^{+}$or $p K^{0}$ ), and those based on a "diquark-diquark- $\vec{s}$ " configuration. Below is a summary of the pentaquark interpolating fields we use that couple to spin- $1 / 2$ states,

$$
\begin{gathered}
\chi_{N K}= \\
\frac{1}{\sqrt{2}} \epsilon^{a b c}\left(u^{T a} C \gamma_{5} d^{b}\right)\left\{u^{c}\left(\bar{s}^{e} i \gamma_{5} d^{e}\right) \mp(u \leftrightarrow d)\right\}, \\
\chi_{\widetilde{N K}}= \\
\frac{1}{\sqrt{2}} \epsilon^{a b c}\left(u^{T a} C \gamma_{5} d^{b}\right)\left\{u^{e}\left(\bar{s}^{e} i \gamma_{5} d^{c}\right) \mp(u \leftrightarrow d)\right\}, \\
\chi_{P S}=\epsilon^{a b c} \epsilon^{a e f} \epsilon^{b g h}\left(u^{T e} C d^{f}\right)\left(u^{T g} C \gamma_{5} d^{h}\right) C \bar{s}^{T c}, \\
\chi_{S S}=\frac{1}{\sqrt{2}} \epsilon^{a b c}\left(u^{T a} C \gamma_{5} d^{b}\right)\left(u^{T c} C \gamma_{5} d^{e}\right) C \bar{s}^{T e} .
\end{gathered}
$$

Here $u, d, \bar{s}$ correspond to up, down and antistrange quarks respectively, the letters $a-h$ refer to the colour of the quarks and $C$ is the charge conjugation matrix. For $\chi_{N K}$ and $\chi_{\widetilde{N K}}$, the and + corresponds to the isospin $I=0$ and 1 channels, while $\chi_{P S}$ and $\chi_{S S}$ access isoscalar and isovector states respectively. In addition to these fields we have also considered an $N K^{*}$-type interpolating field that combines the standard spin$1 / 2$ nucleon interpolating field with a spin- $1 K^{*}$ meson interpolating fields. This pentaquark interpolator couples to both spin- $1 / 2$ and spin-3/2 states. In Sakurai's notation

$$
\begin{aligned}
& \chi_{N K^{*}}^{\mu}= \\
& \quad \frac{1}{\sqrt{2}} \epsilon^{a b c}\left(u^{T a} C \gamma_{5} d^{b}\right)\left\{u^{c}\left(\bar{s}^{e} i \gamma^{\mu} d^{e}\right) \mp(u \leftrightarrow d)\right\},
\end{aligned}
$$

where the - and + corresponds to the isospin $I=0$ and 1 channels, respectively. To project 
states of definite spin from the correlation function extracted with the $\chi_{N K^{*}}^{\mu}$ interpolator we apply the spin projection operators [15],

$$
\begin{aligned}
P_{\mu \nu}^{\frac{3}{2}}(p) & = \\
g_{\mu \nu} & -\frac{1}{3} \gamma_{\mu} \gamma_{\nu}-\frac{1}{3 p^{2}}\left(\gamma \cdot p \gamma_{\mu} p_{\nu}+p_{\mu} \gamma_{\nu} \gamma \cdot p\right) \\
P_{\mu \nu}^{\frac{1}{2}}(p) & =g_{\mu \nu}-P_{\mu \nu}^{\frac{3}{2}}(p) .
\end{aligned}
$$

To extract $1^{-+}$exotic states we use local interpolating fields, coupling colour-octet quark bilinears to chromo-electric and chromo-magnetic fields. We consider four interpolating fields for the $1^{-+}$exotic,

$$
\begin{aligned}
& \chi_{1}=\bar{q}^{a} \gamma_{4} E_{j}^{a b} q^{b} \\
& \chi_{2}=i \epsilon_{j k l} \bar{q}^{a} \gamma_{k} B_{l}^{a b} q^{b} \\
& \chi_{3}=i \epsilon_{j k l} \bar{q}^{a} \gamma_{4} \gamma_{k} B_{l}^{a b} q^{b} \\
& \chi_{4}=\epsilon_{j k l} \bar{q}^{a} \gamma_{5} \gamma_{4} \gamma_{k} E_{l}^{a b} q^{b} .
\end{aligned}
$$

The interpolating fields which couple large-large and small-small spinor components (i.e $\chi_{2}$ and $\left.\chi_{3}\right)$ provide the strongest signal for the $1^{-+}$state.

\subsection{Simulation parameters}

In our analysis we use a large $20^{3} \times 40$ lattice. Using the mean-field $\mathcal{O}\left(a^{2}\right)$-improved Luscher-Weisz plaquette plus rectangle action [18], the gauge configurations are generated via the Cabibbo-Marinari pseudoheat-bath algorithm with three diagonal $\mathrm{SU}(2)$ subgroups looped over twice. The lattice spacing is $0.128(2)$ $\mathrm{fm}$, determined using the Sommer scale $r_{0}=0.49$ $\mathrm{fm}$. For the fermion propagators, we use the FLIC fermion action [19], an $\mathcal{O}(a)$-improved fermion action with excellent scaling properties providing near continuum results at finite lattice spacing [20]. A fixed boundary condition in the time direction is implemented by setting $U_{t}\left(\vec{x}, N_{t}\right)=0 \forall \vec{x}$ in the hopping terms of the fermion action, and periodic boundary conditions are imposed in the spatial directions. Gaugeinvariant Gaussian smearing [21] in the spatial dimensions is applied at the fermion source at $t=8$ to increase the overlap of the interpolators with the ground states. Eight quark masses are used in the $1^{-+}$calculation, providing $a m_{\pi}=$ $\{0.540,0.500,0.453,0.400,0.345,0.300,0.238$, $0.188\}$. For the pentaquark study we limited our calculations to the six largest quark masses because of large statistical uncertainties. The strange quark mass is taken to be the third largest $(\kappa=0.12885)$ quark mass. This $\kappa$ provides a pseudoscalar mass of $697 \mathrm{MeV}$ which compares well with the experimental value of $\sqrt{2 M_{\mathrm{K}}^{2}-M_{\pi}^{2}}=693 \mathrm{MeV}$, which is motivated by leading order chiral perturbation theory. The error analysis is performed by a second-order, single-elimination jackknife, with the $\chi^{2}$ per degree of freedom obtained via covariance matrix fits. Further details of the fermion action and simulation parameters are provided in Refs. $[19,20]$.

\section{Lattice resonance signature}

A test that has proved useful in studies of nucleon resonances [13-15] is a search for evidence of binding at quark masses near the physical regime. In Fig. 1 we show the spectrum of nucleon and $\Delta$ resonances $[14,15]$. The solid curve is the SWave $N+\pi$ decay channel energy corresponding to the $1 / 2^{-}$and $3 / 2^{-}$states which clearly become bound, i.e. the mass of the resonance becomes less than its decay channel energy, at the quark masses shown. This is what we refer to as the standard lattice resonance signature of binding at quark masses near the physical regime. Note that the $3 / 2^{+}$state also becomes bound as it decays to a P-Wave $N+\pi$ (not shown), which due to finite volume effects is at a higher energy than the S-Wave $N+\pi$. We can calculate the energies of P-Wave decay channels by adding the minimum non-trivial momentum on the lattice, $\frac{2 \pi}{L}$ where $L$ is the spatial dimension of the lattice, to each decay constituent. For the $1^{-+}$the lowest energy decay channel is the $a_{1} \eta^{\prime}$. A summary of pentaquark states and their respective decay channels is presented in Table 1 .

\section{Results}

We begin the discussion of our results with the search for the $\Theta^{+}$pentaquark. In Fig. 2 we present a summary of our most relevant results $[2,3]$. We find that the negative parity states in both spin- $1 / 2$ and spin- $3 / 2$ channels are more massive than their decay channel energies. While 

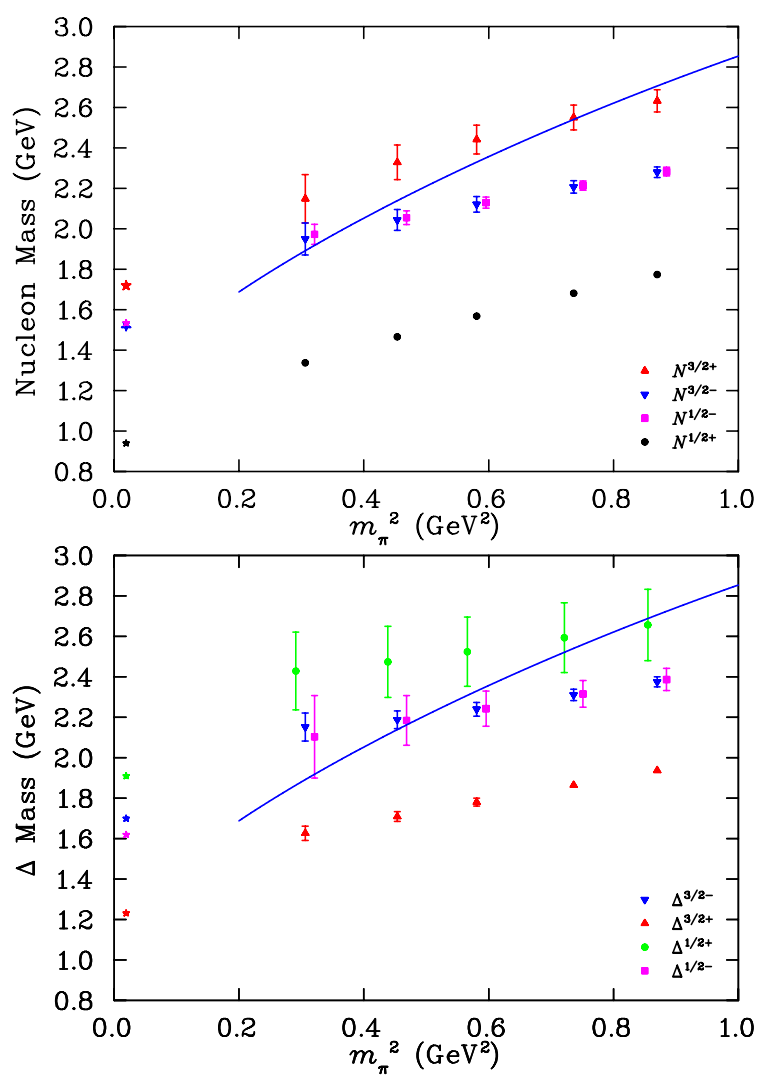

Figure 1. Summary of nucleon resonances $[14,15]$, the solid curve is the mass of the S-Wave $N+\pi$ decay channel corresponding to $1 / 2^{-}, 3 / 2^{-}$parity states.

\section{Table 1}

A summary of pentaquark states and the respective lowest energy decay channels.

\begin{tabular}{|c|c|}
\hline$I\left(J^{P}\right)$ & Decay channel \\
\hline $0,1{\left(\frac{1}{2}^{-}\right)}^{-}$ & $S$-wave $N+K$ \\
\hline $0,1\left(^{+}{ }^{+}\right)$ & $P$-wave $N+K$ \\
\hline $0\left(\frac{3}{2}^{-}\right)$ & $S$-wave $N+K^{*}$ \\
\hline $0\left(\frac{3}{2}^{+}\right)$ & $P$-wave $N+K$ \\
\hline $1\left(\frac{3}{2}^{-}\right)$ & $S$-wave $\Delta+K$ \\
\hline $1\left(\frac{3}{2}^{+}\right)$ & $P$-wave $N+K$ \\
\hline
\end{tabular}

this does not exclude the possible existence of the $\Theta^{+}$in these channels, it has been shown $[8,12]$ that the $I\left(J^{P}\right)=0\left(1 / 2^{-}\right)$and $0\left(3 / 2^{-}\right)$are scattering states, where the masses of these states are larger than their respective free decay channel energies because of finite volume effects. However in the $0\left(3 / 2^{+}\right)$channel the standard lattice resonance signature is seen. Further, quark counting rules imply that the mass splitting between the $\Theta^{+}$and its decay channel should vanish (relative to the quark mass) in the limit of large quark masses, which we observe. Therefore we find that the $0\left(3 / 2^{+}\right)$channel is a promising candidate for future research. Further, because of large statistical errors, evidence of binding cannot be ruled out in the $0\left(1 / 2^{+}\right)$.

In Fig. 3 we present the $1^{-+}$exotic mass extracted with the $\chi_{2}$ interpolator, the $a_{1} \eta^{\prime}$ decay channel energy, at each quark mass, and the $\pi_{1}(1600)$ experimental candidate. At the four largest quark masses the standard lattice resonance signature of binding quark masses near the physical regime is observed. In the approach to the large quark mass limit, quark counting rules imply that the mass splitting should become larger, which we observe. Further we are satisfied that at the lighter quark masses the $\chi_{2}$ interpolator is not accessing the $a_{1} \eta^{\prime}$ decay channel as no evidence of negative metric contributions is observed in our correlation functions [7]. Therefore we tentatively identify this state as the $1^{-+}$exotic meson. We extrapolate to the chiral limit by fitting the function,

$$
m_{1-+}=a_{0}+a_{2} m_{\pi}^{2}+a_{4} m_{\pi}^{4},
$$

to the $1^{-+}$mass in Fig. 3 and recover a mass of $1.74(25) \mathrm{GeV}$ which is consistent to within errors of the $\pi_{1}(1600)$ experimental candidate. Systematic errors associated with the naive extrapolation are estimated to be $\pm 50 \mathrm{MeV}[7]$.

\section{Conclusions}

We have completed a comprehensive search in quenched lattice QCD for the $\Theta^{+}$pentaquark and the $1^{-+}$exotic meson.

We find evidence of the standard lattice signature in the spin- $3 / 2$ isoscalar positive parity chan- 

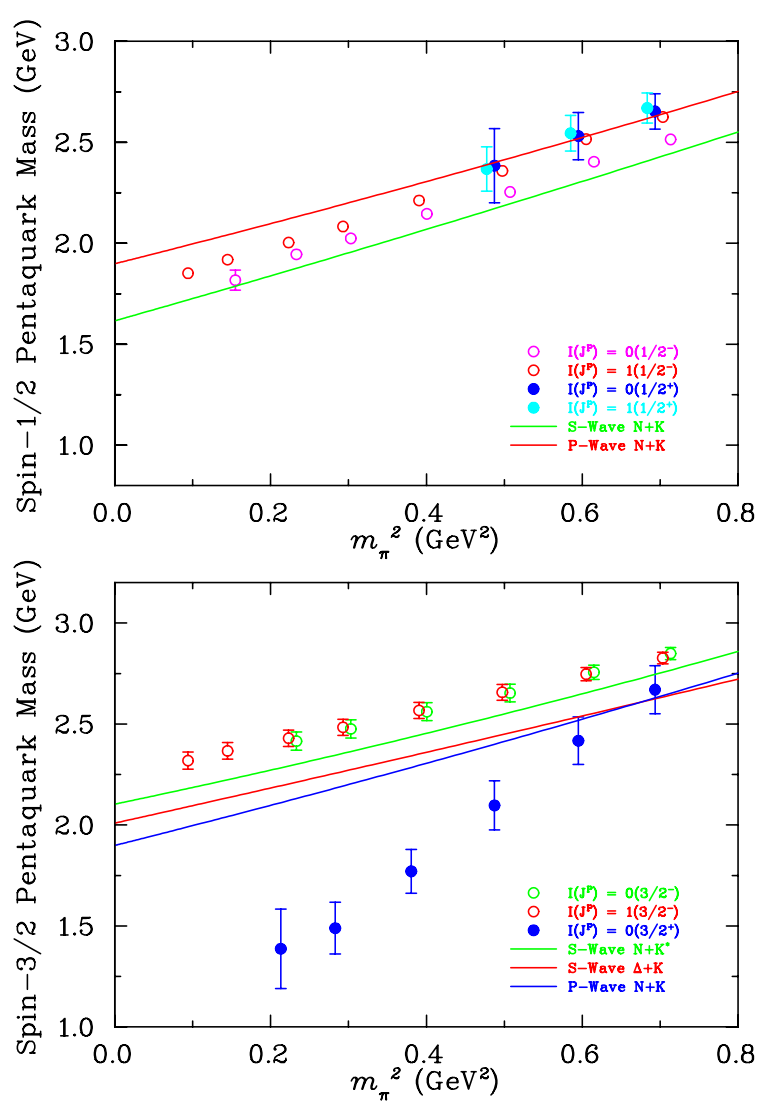

Figure 2. A summary of results extracted with spin- $1 / 2$ and spin- $3 / 2$ pentaquark interpolators and their respective decay channel energies.

nel, making it a promising candidate for future research. An analysis of the volume dependence of this signal is required to determine if this signature implies the existence of the $\Theta^{+}$pentaquark.

With the $\chi_{2}$ hybrid meson interpolator we extract a state which we tentatively identify as a $1^{-+}$exotic meson. Extrapolation to the chiral limit yields, for the first time, a mass that is consistent with the $\pi_{1}(1600)$ experimental candidate.

\section{REFERENCES}

1. K. Hicks, hep-ex/0501018.

2. B. G. Lasscock et. al., Phys. Rev. D72

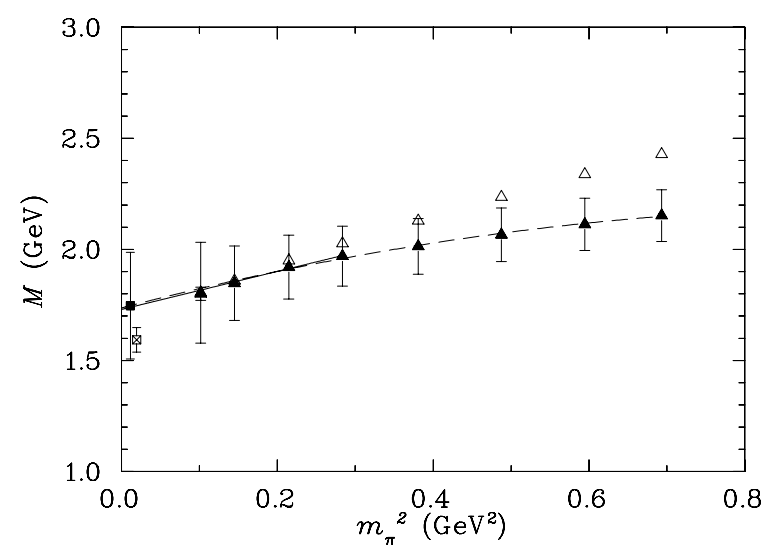

Figure 3 . The $1^{-+}$mass extracted with the $\chi_{3}$ interpolator (closed triangles), the $a_{1} \eta^{\prime}$ decay channel (open triangles) and the $\pi_{1}(1600)$ experimental candidate (square).

(2005) 014502 [hep-lat/0503008].

3. B. G. Lasscock et. al., Phys. Rev. D72 (2005) 074507 [hep-lat/0504015].

4. Particle Data Group Collaboration, S. Eidelman et. al., Phys. Lett. B592 (2004)

5. $\mathbf{E 8 5 2}$ Collaboration, M. Lu et. al., Phys. Rev. Lett. 94 (2005) 032002 [hep-ex/0405044].

6. A. R. Dzierba, R. Mitchell, A. P. Szczepaniak, M. Swat and S. Teige, hep-ex/0502022.

7. J. N. Hedditch et. al., Accepted for publication in Phys. Rev. D (2005) hep-lat/0509106.

8. N. Mathur et. al., Phys. Rev. D70 (2004) 074508 [hep-ph/0406196].

9. C. Alexandrou and A. Tsapalis, hep-lat/0503013.

10. N. Ishii et. al., Phys. Rev. D71 (2005) 034001 [hep-lat/0408030].

11. N. Ishii et. al., hep-lat/0410022.

12. N. Ishii, T. Doi, Y. Nemoto, M. Oka and H. Suganuma, Phys. Rev. D72 (2005) 074503 [hep-lat/0506022].

13. D. B. Leinweber, W. Melnitchouk, D. G. Richards, A. G. Williams and J. M. Zanotti, 
Lect. Notes Phys. 663 (2005) 71-110.

14. W. Melnitchouk et. al., Phys. Rev. D67 (2003) 114506 [hep-lat/0202022].

\section{CSSM Lattice Collaboration}

Collaboration, J. M. Zanotti et. al., Phys.

Rev. D68 (2003) 054506 [hep-lat/0304001].

16. S. Sasaki, T. Blum and S. Ohta, Phys. Rev. D65 (2002) 074503 [hep-lat/0102010].

17. QCDSF Collaboration, M. Gockeler et. al., Phys. Lett. B532 (2002) 63-70 [hep-lat/0106022].

18. M. Luscher and P. Weisz, Commun. Math. Phys. 97 (1985) 59.

19. CSSM Lattice Collaboration Collaboration, J. M. Zanotti et. al., Phys. Rev. D65 (2002) 074507 [hep-lat/0110216].

20. J. M. Zanotti, B. Lasscock, D. B. Leinweber and A. G. Williams, Phys. Rev. D71 (2005) 034510 [hep-lat/0405015].

21. S. Gusken, Nucl. Phys. Proc. Suppl. 17 (1990) 361-364. 